\title{
Stochastic generation scheduling with variable renewable generation: methods, applications, and future trends
}

\author{
ISSN 1751-8687 \\ Received on 5th August 2018 \\ Revised 28th November 2018 \\ Accepted on 2nd January 2019 \\ E-First on 4th April 2019 \\ doi: 10.1049/iet-gtd.2018.6331 \\ www.ietdl.org
}

\author{
Wen-Shan Tan ${ }^{1}$, Mohamed Shaaban ${ }^{2} \bowtie$, Mohd Zainal Abidin Ab Kadir ${ }^{3}$ \\ ${ }^{1}$ School of Engineering, Monash University Malaysia, Jalan Lagoon Selatan, 46150 Bandar Sunway, Selangor, Malaysia \\ ${ }^{2}$ Department of Electrical \& Electronic Engineering, Faculty of Engineering, Universiti Malaysia Sarawak, 94300 Kota Samarahan, Sarawak, \\ Malaysia \\ ${ }^{3}$ Institute of Power Engineering, Universiti Tenaga Nasional, 43000 Kajang, Selangor, Malaysia \\ ¿-mail: mshaaban@unimas.my
}

\begin{abstract}
One of the most intricate optimisation problems in power systems is generation scheduling. It determines the schedule and dispatch of electrical power generation to meet the load demand under various technical and operating constraints. Generation scheduling is a vivid problem, particularly in recent years, due to the aggressive integration of renewable energy, with stochastic nature, into power grids. As the literature size has swollen substantially in the past several years, this study critically looks into uncertainty modelling and the formulation of various techniques that were implemented in stochastic optimisation-based generation scheduling. The strengths and weaknesses of existing methods are fully exposed. Market operation policies that significantly affect the scheduling of renewable energy generation in different timescales are elaborated. Potential applications and future trends in terms of modelling, computational performance, and incorporation of flexibility and resilience notions are thoroughly discussed.
\end{abstract}

\section{Introduction}

Since the birth of generation scheduling (GS), there have been two waves of revolution, as far as the research and application in the industry is concerned. Mixed integer linear programming (MILP) stood its ground among deterministic GS approaches such as dynamic programming, priority list, and Lagrangian relaxation, and became the state-of-the-art solution approach in the first wave of revolution $[1,2]$. The second wave of revolution has underscored transforming conventional deterministic approaches to stochastic GS ones. This trend was mainly propelled by the emerging challenges to power system operation in the past three decades, including; deregulation of electricity grids, integration of renewable energy generation (REG), and increased demand of system reliability, flexibility and resilience.

GS is often utilised by independent system operators (ISOs) in deregulated electricity markets, for cost minimisation, in the dayahead market clearing, reliability assessment, and intra-day or realtime operations. GS problem is proven to be non-deterministic polynomial-time hard (NP-hard) [1], in which the computation time exponentially increases along with the size of the problem. This is due to the use of binary variables to represent the generation units' commitment (ON/OFF status), and the combination of a large number of operating constraints.

Various approaches were proposed for the deterministic GS, such as conventional dynamic programming [3-7] and priority list [8-11]. These approaches were under continuous research \& development until they were in full swing over the years to engender the current, most frequently utilised MILP approaches. The general formulation of a deterministic MILP-based GS model, which covers an objective function to minimise total production cost (fuel cost, start-up cost, and shut-down cost) and various technical constraints (generation, power balance, minimum up/ down time, ramping, spinning reserve, and transmission constraints) have been presented and discussed in [12, 13].

Flexibility is the ability of a system to act in response to variability and uncertainty of demand and supply. With the growing percentage of electricity generated by REG in power grids, introducing significant uncertainty and variability into the system, the intertwined relation between renewables and flexibility has drawn considerable attention. Successful integration of REG into the grid brings about a new operation paradigm, per se, which involves unprecedented operation scenarios, as well as diverse technical and organisational challenges that require major coordination. These challenges could be tackled by new customised market designs and/or technology-agnostic operational practices.

A number of measures were proposed to improve power system planning and operation with REG, such as utilisation of flexible generation, energy storage, and demand response. In addition to the above measures, a stochastic view to replacing current deterministic GS models was proposed as a promising approach to improve the system's reliability. Stochastic programming can incorporate uncertainty of coefficients or parameters as random variables with an assumed probability distribution or capture the uncertainty through scenario generation. Although stochastic optimisation such as full stochastic programming (FSP) is a fullblown technology in the modelling of uncertainty in various research areas [14], nonetheless, its application to the NP-hard GS problem still contains challenging barriers and bottlenecks. ISOs and generation companies are concerned about the complexity of FSP, which leads to higher computational requirements and infeasibility from the standpoint of practical realisation in the electricity market.

In this study, state-of-the-art applications of stochastic optimisation methods in GS are surveyed and scrutinised. On top of that, the paper reviews the prospective applications and research trends of stochastic GS in electricity markets. Furthermore, it sheds light on power system flexibility and resilience issues. Owing to the large size of the literature, along with the intent to focus on the REG integration in short-term stochastic GS, discussions in this paper are categorically limited to the following scope:

i. Formulation and solution of stochastic GS models, rather than deterministic GS models with supplementary constraints (e.g. reserve requirement constraints), which are based on probabilistic forecasts.

ii. Short-term GS (day-ahead and hourly-ahead) in preference to longer-term GS (weekly, yearly, and seasonally). 